\title{
Micro-liquid flow sensor
}

\author{
Theo S J Lammerınk, Niels R Tas, Miko Elwenspoek and Jan H J Fluitman \\ MESA Research Institute, University of Twente, $P O$ Box 217, 7500 AE Enschede (Netherlands)
}

\begin{abstract}
A simple to realise micro-liquid flow sensor with high sensitivity is presented The sensor is based on well known thermal anemometer principles An analytical model for the sensor behaviour applicable for gas/liquid fluids is presented The realisation process of the sensor is described Model and experimental results agree well The sensor is simple to integrate with other micro-liquid handling components such as pumps, mixers, etc
\end{abstract}

\section{Introduction}

Recently there is a growing interest in the research on micro-liquid handling systems [1] One of the basic components in liquid handling systems is the microliquid flow sensor Liquid flow sensors described in the literature make use of the 'time-of-flight' flow-sensing technique $[2,3]$

Other micromachined flow sensors which are realised in or close to the flow channel are designed for sensing gas flow [4-6] They make use of a heater the temperature of which is a function of the heat carried away by the medium Other fluid-sensing technques are described in ref 7 The dissipation in the heater causes a temperature distribution in the flow channel, which is modulated by the medium Flow sensors, which make use of more than one temperature sensor to get a signal, that is related to the flow, are designed to sense the flow of gaseous media $[5,8,9]$

For an optimal design one can make use of a numercal approach [10] For a good understanding and for obtaining design rules it is advantageous to derive an analytical model

\section{Model}

Figure 1 illustrates the principle of the flow sensor Three resistors are located in the middle of the flow channel Heat is dissipated in the middle resistor called the heater $(\mathrm{H})$ The resulting temperature distribution is sensed with two temperature sensitive resistors $T_{1}$ and $T_{2}$ symmetrically located near the heater One resistor is located upstream relative to the heater and one resistor is located downstream The heater as well as the sensing resistors are located on supporting beams which cross the flow channel in the $x-y$ plane (see Figs 7 and 8)

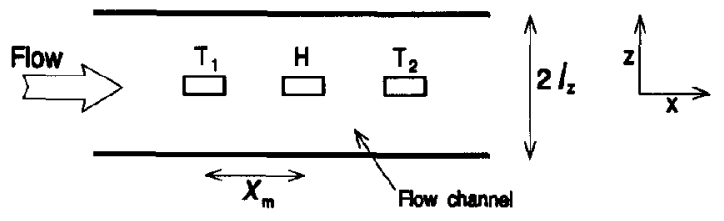

Fig 1 The flow is measured by its influence on the temperature distribution in the sensor resulting from heat generation in the heater $\mathrm{H} \quad \mathrm{T}_{1}$ (upstream) and $\mathrm{T}_{2}$ (downstream) are temperature sensors

At zero flow rate, no convection exists in the flow channel, and the heat generated by the heater will be transferred only by the axial and tangential diffusion through the fluid and by the conduction through the heater and sensor support to the flow channel walls

\section{Heater temperature (flow $=0$ )}

A sumple model for the conduction in the $z-y$ plane perpendicular to the flow channel axis is based on lumped elements for the thermal conductivity present (see Fig 2) The heat is homogeneously dissipated along the resistor in the $y$ direction The conduction in the beam is modelled with $G_{\mathrm{b}}$ and that in the fluid with $G_{\mathrm{f}}$

If we model the channel cross section with $n$ lumped elements (in the beam), the different lumped element values are given in the following equations

$$
\begin{aligned}
& \sum_{t=1}^{n-1} P_{\mathrm{t}}=P_{\mathrm{t}} \quad P_{\mathrm{t}}=\frac{P_{\mathrm{t}}}{(n-1)} \\
& G_{\mathrm{b}}=(n-1) G_{\mathrm{b} 0} \quad G_{\mathrm{f}}=\frac{G_{\mathrm{f}}}{(n-1)}
\end{aligned}
$$

where $P_{\mathrm{t}}, G_{\mathrm{b} 0}$ and $G_{\mathrm{f}}$ are the total dissipated power, the beam conductivity and the fluid conductivity, respectively $P_{1}, G_{\mathrm{b}}$ and $G_{\mathrm{f}}$ are the node and the element values, respectively 

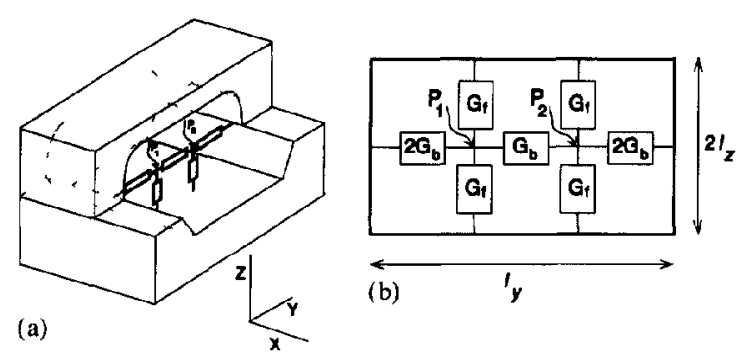

(c)
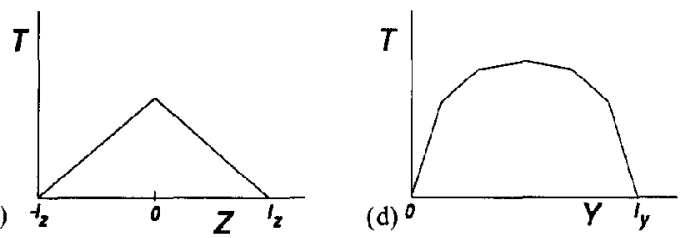

Fig 2 Lumped element model of $y-z$ plane of heater (a) Flow channel with heater resistor divided into three parts (b) $y-z$ plane with flud and beam conductivities (c) Temperature profile in $z$ direction (d) Temperature profile in $y$ direction (beam divided into 6 elements)

In this model the temperature distribution in the fluid is assumed to be linear in the $z$ direction (see $F_{1} g$ 2(c)) The temperature distribution in the $y$ direction is given in Fig 2(d) The temperature $T(y)$ depends on the relative conductivity of the fluid involved and the beam material With the model it can be shown that, at least for liquids, the heat conduction through the beam can be omitted For the convection model it is assumed that $T(y)=$ constant

Due to the geometry of the channel, there is little heat transport to the side walls compared to the transport to the floor and celling In our further analysis we concentrate on the heat transport in the $x$ and $z$ directions

\section{Convection}

In a quasi-static situation, the incoming heat at a certain point in the fluid must be equal to the outgoing heat The heat is transported either by conduction in the fluid and/or supporting beams or by convection through the thermal mass of the fluid Ultimately, the heat is transported to the walls of the flow channel A heat balance equation results in a differential equation for $T$ in $x$ The temperature profile in the $y$ and $z$ directions is assumed to be constant and linear, respectively (see Fig 2(c))

With $A$ the cross section area of the flow channel ( $A=l_{y} 2 l_{y}$ ), $\rho$ the fluid density, $c$ the fluid heat capacity (at constant pressure), $v$ the fluid velocity and $\kappa$ the fluid thermal conductivity we find

$$
A \kappa \frac{\partial^{2} T}{\partial x^{2}}-A \rho c v \frac{\partial T}{\partial x}-\kappa \frac{2 l_{2}}{l_{z}} T=0
$$

or

$D \frac{\partial^{2} T}{\partial x^{2}}-v \frac{\partial T}{\partial x}-g D T=0$

with $D=\kappa / \rho c$ the thermal diffusivity of the fluid and $g=1 / l_{z}^{2}$ Equation (2) is linear in $T$ Solving the differential equation for a heater length of $2 J$, a heater power $P$ and using the boundary conditions, $\lim x \rightarrow$ $\pm \infty T(x)=0$, the following temperature distribution results

$\begin{array}{ll}x<-L & T(x)=T_{0} \exp \left(\lambda_{1}(x+L)\right) \\ -L<x<L & T(x)=T_{0} \\ x>L & T(x)=T_{0} \exp \left(\lambda_{2}(x-L)\right)\end{array}$

with

$\lambda_{12}=\frac{1}{2 D}\left\{v \pm \sqrt{v^{2}+4 g D^{2}}\right\}$

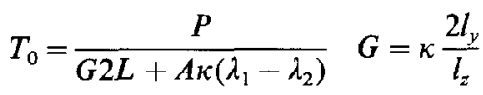

$2 L$ is the heater 'length' ( $x$ direction) In eqn (3) the e1genvalues $\lambda_{1}$ and $\lambda_{2}$ depend on the flow velocity $v$ Above the heater only heat conduction in the direction perpendicular to the flow direction is assumed (heat conduction term $G 2 L$ in eqn (3c)) At the heater edges, heat is injected in the flund stream upstream (conduction term $\left.A \kappa \lambda_{1}\right)$ and downstream $\left(-A \kappa \lambda_{2}\right)$ The temperature distribution for three different flow velocities is given in Fig 3

The temperature at the two sensor sides, upstream (at $x=x_{\mathrm{m}}$ ) and downstream (at $x=-x_{\mathrm{m}}$ ) and the temperature difference $\Delta T$ are given by

$T_{\mathrm{d}}=T_{0} \exp \left(\lambda_{2}\left(x_{\mathrm{m}}-L\right)\right)$

$T_{\mathrm{u}}=T_{0} \exp \left(\lambda_{1}\left(-x_{\mathrm{m}}+L\right)\right)$

$\Delta T=T_{\mathrm{d}}-T_{\mathrm{u}}$

$\Delta T=T_{0}\left\{\exp \left(\lambda_{2}\left(x_{\mathrm{m}}-L\right)\right)-\exp \left(\lambda_{1}\left(-x_{\mathrm{m}}+L\right)\right)\right\}$

The temperature $T_{\mathrm{u}}, T_{\mathrm{d}}, \Delta T$ and the heater temperature $T_{\mathrm{h}}$ are given in Fig 4

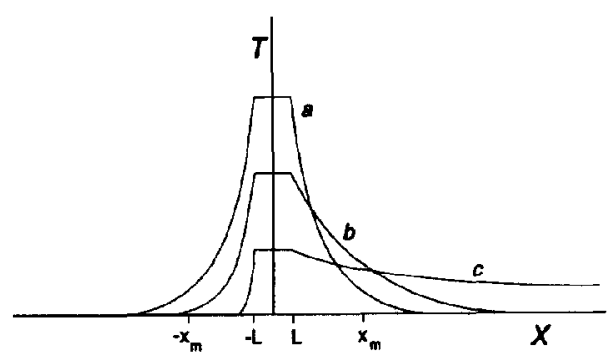

Fig 3 Temperature distribution in the middle of the channel as a function of the position $x$ The heater is from $x=-L$ to $x=L$ (a) $v=0$, (b) $v=0001$, (c) $v=001$ 


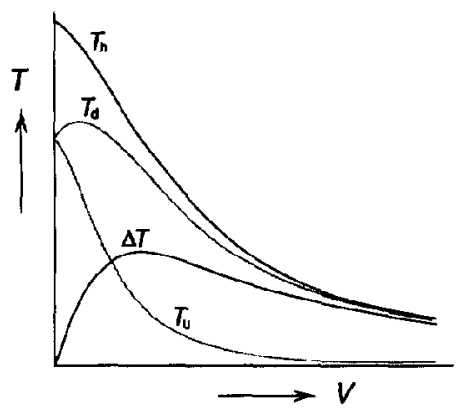

Fig 4 Sensor temperatures as function of the flow velocity $v T_{\mathrm{h}}$ is the heater temperatrure, $T_{\mathrm{u}}$ and $T_{\mathrm{d}}$ the upstream and downstream sensor temperature, respectively, and $\Delta T$ the temperature difference between the downstream sensor and upstream sensor

\section{Model results}

\section{Sensitwity}

For relative low flow velocities the flow sensor has an almost linear flow dependence The sensitivity $S$ at $v=0$ is defined as the derivative of $\Delta T$ with respect to $v(S=\partial \Delta T / \partial v)$

$\Delta T=S v$

From eqns (3) and (4) and assuming $L=0$ we find

$S=\frac{P}{4 \kappa l_{y}} \frac{x_{\mathrm{m}}}{D} \exp \left(-x_{\mathrm{m}} / l_{z}\right)$

with $P$ the dissipated power in the heater, $\kappa$ the fluid heat conductivity, $l_{y}$ the channel width, $x_{m}$ the measurement distance, $l_{z}$ half the channel height and $D$ the fluid thermal diffusivity The sensitivity as a function of the quotient $x_{\mathrm{m}} / l_{z}$ is given in Fig 5 The sensitivity of the flow sensor has a maximum for $x_{\mathrm{m}}=l_{z}$ From eqn (6) and assuming that $x_{\mathrm{m}}=l_{z}$, this maximum sensitivity $S_{\max }$ is

$S_{\max }=0092 \frac{l_{z}}{l_{y}} \frac{P}{\kappa D}$

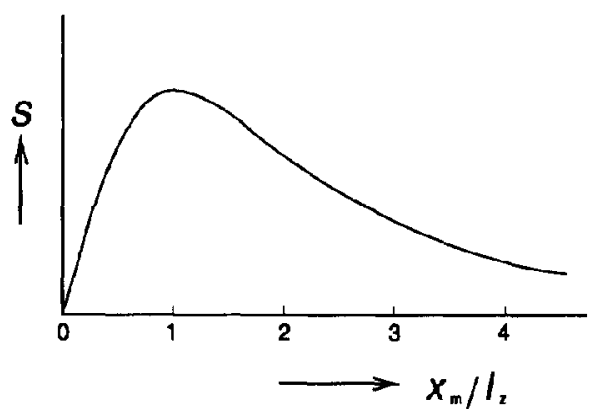

Fig 5 Sensor sensitivity $S$ as a function of the geometrical factor $x_{\mathrm{m}} / l_{z}$
For a flow sensor, sensing water flow with $l_{y}=$ $1000 \mu \mathrm{m}, l_{z}=250 \mu \mathrm{m}, \kappa=06 \mathrm{~W} / \mathrm{mK}, D=14 \times 10^{-7}$ $\mathrm{m}^{2} / \mathrm{s}$ and $10 \mathrm{~mW}$ dissipation in the heater we find $S=27 \times 10^{3} \mathrm{~K} /(\mathrm{m} / \mathrm{s})$

When the heater and the temperature sensors are modelled as distributed elements, the global sensor properties do not change For venfication, the behaviour of a sensor with the heater (length $l_{h}=2 L$ ) and the sensor up/downstream (length $l_{\mathrm{s}}$ of sensors is also $2 L$ ) divided in five pieces is numerically evaluated This is relatively easy because eqns (2) and (3) are linear in $T$ The calculations show a decrease of the sensitivity $S$ of about $20 \%$

\section{Flow range}

For relative low flow velocities the temperature difference $\Delta T$ between the upstream and downstream temperature sensors is an almost linear function of $v$ (see eqn (5) and Fig 4) However, at a certain flow, the temperature difference reaches a maximum and for higher flow velocities $\Delta T$ is even lower This is caused by a decrease of the heater temperature (see also Fig 4)

The flow for which this maximum is reached depends on $x_{\mathrm{m}}, D, A, g$ and $\kappa$ From eqn (3b) we can see that the eigenvalues $\lambda_{12}$ show a changing flow dependence around

$v^{2}=4 g D^{2}$

The flow velocity, which is found from eqn (8) is called the turn-over flow velocity $v_{\text {to }}$

$v_{\mathrm{to}}=\frac{2 D}{l_{z}}$

For example with 2-propanol as a fluid $\left(D=696 \times 10^{-8} \mathrm{~m}^{2} / \mathrm{s}\right)$ and $l_{z}=250 \mu \mathrm{m}$ we find $v_{\text {to }}=056 \times 10^{-3} \mathrm{~m} / \mathrm{s}$ The turn-over flow velcolty $v_{\text {to }}$ determines the useful flow range of the flow sensor

\section{Fluzd dependence}

The sensor characteristics are strongly influenced by the thermal diffusivity of the fluid involved From eqn

(2) it is seen that the temperature distribution (see also Fig 4) is controlled by the ratio of the flow velocity and the thermal diffusivity $v / D$ A liquid fluid like 1sopropanol has $D=696 \times 10^{-8} \mathrm{~m}^{2} / \mathrm{s}$ For a gaseous fluid (arr), $D=186 \times 10^{-5} \mathrm{~m}^{2} / \mathrm{s}$ The velocity scale, as used in Fig 4, differs by a factor 250 for these two fluids

\section{Realisation}

The realisation process of the flow sensor is shown in Fig 6 On a $300 \mu \mathrm{m}\langle 100\rangle$ Si wafer a $1 \mu \mathrm{m}$ LPCVD SiN layer is deposited (Fig 6(a)) The deposition 


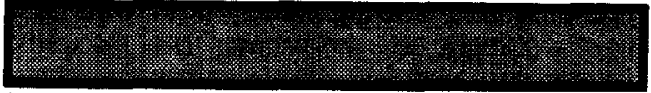

(a)

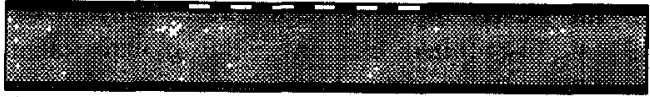

(b)

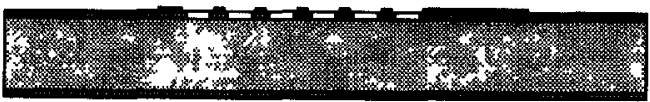

(c)

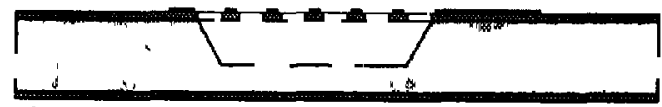

(d)

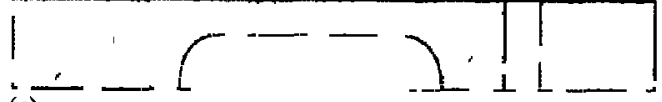

(e)

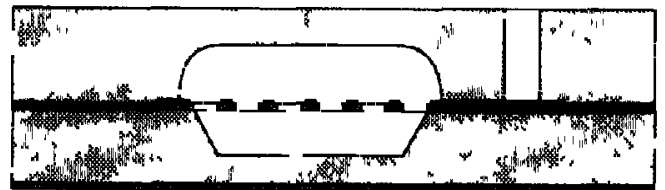

(f)

F1g 6 Process sequence for realısing the flow sensor

parameters are such that there is no mechanical stress in the SiN layer ('stress free') The SIN layer is patterned with the anisotropically Si-etch mask needed for the flow channel (Fig 6(b)) The flow channel is aligned parallel to the [110] crystal direction The $\mathrm{SiN}$ beams across the channel have a width of $40 \mu \mathrm{m}$ and are oriented in the [100] crystal direction The beams cross each other at a heart to heart distance of $200 \mu \mathrm{m}$ Then the metal layer with the resistor pattern is applied A $200 \mathrm{~nm} \mathrm{CrAu}$ layer is deposited and patterned (lift-off) The $30 \mathrm{~nm}$ chromium layer is applied for an optımal adhesion of the $\mathrm{Au}$ layer The flow channel is (anisotropically) etched in a $\mathrm{KOH}$ solution at $70^{\circ} \mathrm{C}$. The cross section of the flow channel is an inverse trapezold which is $1000 \mu \mathrm{m}$ wide at the top and has a depth of $250 \mu \mathrm{m}$ In the Pyrex wafer the flow channel is isotropically etched in an HF solution at room temperature with a sputtered polysilicon layer as etch mask The feed troughs $(\phi=400 \mu \mathrm{m})$ for the needed electrical contacts are made by drilhng (Fig 6(e)) Finally the Pyrex wafer and the structured $S_{1}$ wafer are anodically bonded $\left(1250 \mathrm{~V}, 450^{\circ} \mathrm{C}\right)$ The high voltage needed in the bonding process is due to the $(1 \mu \mathrm{m}) \mathrm{S}$ IN layer on top of the silicon

Due to the straightforward realisation process the flow sensor can easily be integrated with other microliquid handling components as pumps [11] and mixers [12]

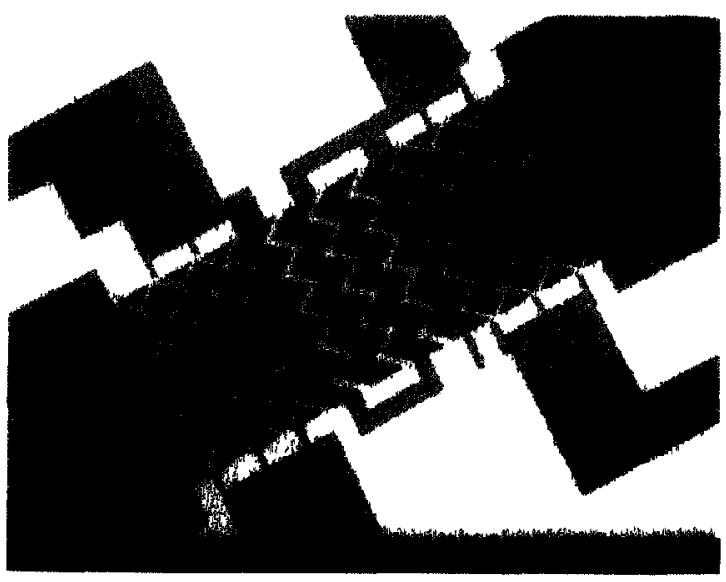

Fig 7 SEM photograph of flow sensor The channel width is $1 \mathrm{~mm}$

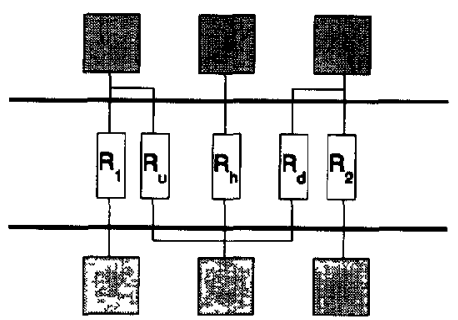

Fig 8 The electrical connection inside the flow sensor $R_{\mathrm{u}}$ and $R_{\mathrm{d}}$ are the sensing resistors upstream and downstream, respectively $R_{\mathrm{h}}$ is the heating resistor Resistors $R_{1}$ and $R_{2}$ are implemented in order to obtain a '4-point' measurement avoiding parasitic series resistances

The realised sensors (see $F_{1} g$ 7) are wired according to Fig 8 Additional contacts are implemented to make an accurate resistance measurement possible

\section{Expenments}

The sensors are charactensed with the measurement circuit given in Fig 9 The temperature measuring

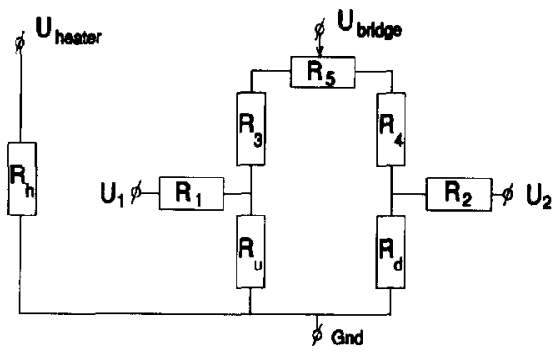

Fig 9 Sensor measurement circuit Nominal resistor values are $R_{\mathrm{h}}=800 \Omega, R_{\mathrm{u}}, R_{\mathrm{d}}=3300 \Omega, R_{1}, R_{2}=725 \Omega, R_{3}, R_{4}=3300 \Omega$, $R_{\mathrm{s}}=100 \Omega$ 
resistors are placed in a bridge configuration Additional resistors in the flow sensor make it possible to compensate for contact series resistances

The temperature coefficient $T_{\mathrm{c}}$ of the sensing resistors is measured in a temperature controlled furnace In the range from 20 to $50^{\circ} \mathrm{C}$ we found $T_{\mathrm{c}}=15 \times 10^{-5} /{ }^{\circ} \mathrm{C}$ This temperature coefficient is lower than expected A possible cause is the (unwanted) annealing effect of the anodic bonding process on the CrAu metal layers After the bond step the impedance of the resistors showed to be a factor of 20 higher than the value before the bond process

The dissipation in the sensor resistors is so small that they do not significantly modify the temperature distribution resulting from the dissipation in the heater resistor $R_{\mathrm{h}}$ and the flund flow

With $U_{\mathrm{b}}$ as the bridge voltage and assuming that $R_{3}=R_{4}, R_{\mathrm{u}}=R_{\mathrm{d}}$, the relation between the temperature $\Delta T$ and the output voltage $\Delta U$ of the sensor bridge is given

$\Delta U=U_{2}-U_{1}-U_{\mathrm{b}} \frac{R_{\mathrm{u}}}{\left(R_{3}+R_{\mathrm{u}}\right)^{2}} T_{\mathrm{c}} \Delta T$

With $U_{\mathrm{b}}=3 \mathrm{~V}, R_{3}=R_{\mathrm{u}}$ and a measured temperature coefficient $T_{\mathrm{c}}=15 \times 10^{-5} 1 / \mathrm{K}$, the bridge sensitivity $S_{\mathrm{b}}=13 \times 10^{-5} \mathrm{~V} / \mathrm{K}$

Flow velocities are generated with syringe-pumps and have been varied in the range $0-2 \mathrm{~m} / \mathrm{s}$ Measurements are carned out with water and 2-propanol as liquid fluids and with air as gaseous fluid Fluid properties are

TABLE 1 Properties of used fluids

\begin{tabular}{lllll}
\hline Flund & $\begin{array}{l}\kappa \\
(\mathrm{W} / \mathrm{m})\end{array}$ & $\begin{array}{l}\rho \\
\left(\mathrm{kg} / \mathrm{m}^{3}\right)\end{array}$ & $\begin{array}{l}c \\
\left(10^{3} \mathrm{~J} / \mathrm{kgK}\right)\end{array}$ & $\begin{array}{l}D \\
\left(\mathrm{~m}^{2} / \mathrm{s}\right)\end{array}$ \\
\hline 2-Propanol & 0141 & 785 & 285 & $696 \times 10^{-8}$ \\
Water & 06 & 998 & 418 & $14 \times 10^{-7}$ \\
Arr & 0024 & 129 & 10 & $186 \times 10^{-5}$ \\
\hline
\end{tabular}

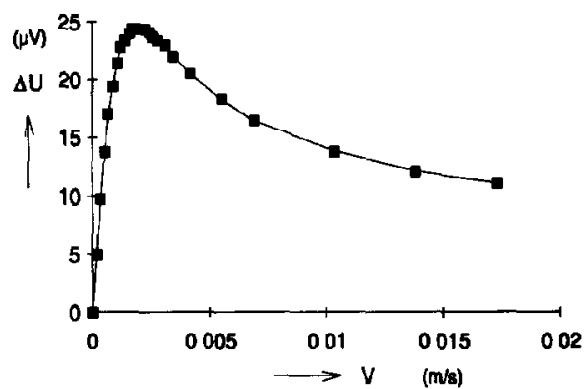

Fig 10 Flow sensor output with water as fluid $W_{1}$ th a used flow channel of $1000 \mu \mathrm{m} \times 500 \mu \mathrm{m}$, a flow velocity $v=20 \mathrm{~mm} / \mathrm{s}$ is equal to a volume flow of $06 \mathrm{ml} / \mathrm{min}$ For water $\kappa=06 \mathrm{~W} / \mathrm{mK}$, $D=14 \times 10^{-7} \mathrm{~m}^{2} / \mathrm{s}$ given in Table 1 The sensor output signals as a function of the applied flow velocity show a typical result as given in Fig 10

\section{Discussion}

The measurement result shown in Table 1 is qualitatively similar to the model results shown in Fig 4 For low flow velocities the output signal is linear with $v$ (see eqn (6)) At a certain flow velocity, the output signal reaches a maxımum, the turn-over flow velocity $v_{t 0}$ (see eqn (9)) For higher flow velocities the sensor output signal decreases with increasing $v$ This is due to a significant decrease of the heater temperature (see eqn (3c) and Fig 4)

We did not measure the temperatures $T_{\mathrm{u}}$ and $T_{\mathrm{d}}$ separately as a function of the flow velocity However, the flow velocity dependences of $T_{\mathrm{u}}$ and $T_{\mathrm{d}}$ as predicted by the model (eqn (4) and Fig (4)), are also observed by Johnson and Higash [8] and by Esashı [9] In our model the conductivity to the walls is modelled in a two-dimensional model ( $y-z$ plane) The cffect of convection on the temperature distribution is modelled in the $x-z$ plane Accurate modelling would need a complete three-dimensional approach However it is difficult to build a three-dimensional analytical model

Esash [9] applies two resistors for the flow sensing function instead of the three resistor design we have realised If the resistors of the two resistor design each dissipate the same amount of heat as the heater resistor in our sensor design, the model predicts the same values for $\Delta T$ (eqn (4)) Yet, with the two resistor design it is more difficult to establish a constant temperature mode of operation

The calculated and measured sensitivities $S$ and turnover flow velocities $v_{\text {to }}$ of three fluids (extracted from $\Delta U-v$ graphs as given in Fig 10 ) are given in Table 2

From eqn (6) we see that the reciprocal sensitivity is proportional to the product of the thermal conductivity $\kappa$ and the thermal diffusivity $D$ The sensitivity values for water match well The medsured sensitivity for isopropanol is less than expected However, for the calculated values, given in Table 2, we assume pointtemperature sensors and we assume a uniform (lammar)

TABLE 2 Calculated and measured sensitivities $S$ and turn-over velocities $v_{t o}$

\begin{tabular}{lllll}
\hline Fluid & $\begin{array}{l}S(\mathrm{calc}) \\
(\mathrm{Ks} / \mathrm{m})\end{array}$ & $\begin{array}{l}S \text { (meas) } \\
(\mathrm{Ks} / \mathrm{m})\end{array}$ & $\begin{array}{l}v_{\text {to }}(\mathrm{calc}) \\
(\mathrm{m} / \mathrm{s})\end{array}$ & $\begin{array}{l}v_{\mathrm{to}} \text { (meas) } \\
(\mathrm{m} / \mathrm{s})\end{array}$ \\
\hline 2-Propanol & $17 \times 10^{4}$ & $08 \times 10^{4}$ & $06 \times 10^{-3}$ & $14 \times 10^{-3}$ \\
Water & $02 \times 10^{4}$ & $02 \times 10^{4}$ & $11 \times 10^{-3}$ & $20 \times 10^{-3}$ \\
Alr & $38 \times 10^{2}$ & $03 \times 10^{2}$ & 0148 & 20 \\
\hline
\end{tabular}


fluid flow profile These conditions are not fulfilled in the experiment

The measured sensitivity for arr is about 8 times less than the expected sensitivity based on the model The assumption that the conduction through the supporting beams is negligible, is difficult to hold for gaseous fluds Also the influence of the flow profile, which we assume uniform, has to be investigated further

The turn-over flow velocity $v_{10}$ is the point where the sensor output as function of the flow velocity reaches its maxumum value The calculated and measured values for $v_{10}$ are given in Table 2 The qualitative agreement is good In our model the temperature distribution strongly depends on the parameter $g$ from eqn (2) (see eqns (3b) and (9)) It is assumed that the heat conductivity through the beam is negligible to the heat conductivity through the fluid Also the conduction to the sidewalls should be calculated for

The quantitative agreement between the calculated and expected values for $S$ and $v_{\text {to }} 1 \mathrm{~s}$ the best for water, which has the highest heat conductivity $\kappa$

\section{Conclusions}

A simple micro-liquid flow sensor has been modelled, designed and realised The flow sensor functions in good qualitative agreement with the predictions of an analytical sensor model The model is based on a lumped element approach with some approximations to simplify it It predicts the temperature distribution in the flow sensor and the flow velocity dependence The geometry, the heat conductivity and the thermal diffusivity determine the sensitivity and flow range The design anticipates the application in a flow controller [11] and other fluid handling systems [12]

\section{Acknowledgements}

The authors thank E Berenschot, $\mathbf{R}$ Groothengel (3T, Twente Technology Transfer) and the staff of the MESA-S\&A laboratory for their help in realising the test samples

\section{References}

I F C M van der Pol and J Branebjerg, Micro fludd-handling systems - state of the art and opportunities, Proc ICAR' 91 , 5th Int Conf Advanced Robotics, Ptsa, Italy, June 19-22, 1991, pp 283-290

2 J Branebjerg, O S Jensen, N G Laursen and O Leıstıco, A micromachined flow sensor for measuring small liquid flows, Proc 6th Int Conf Soltd-State Sensors and Actuators (Transducers '91), San Francisco, CA, USA, June 24-27, 1991, pp 41-44

$3 \mathrm{C}$ Yang and $\mathrm{H}$ Søeberg, Monolithic fow sensor for measuring milhlitre per minute liquid flow, Sensors and Actuators $A$, 33 (1992) 143-153

$4 \mathrm{~K}$ Petersen and J Brown, High precisıon, high performance mass-flow sensor with integrated laminar flow microchannels, Proc 3rd Int Conf Solud-State Sensors and Actuators (Transducers '85), Philadelphta, PA, USA, June 7-11, 1985, pp $361-363$

$5 \mathrm{G}$ Wachutka, $\mathrm{R}$ Lenggenhager, D Moser and $\mathrm{H}$ Baltes, Analytical 2D-model of CMOS micromachined gas flow sensors, Proc 6th Int Conf Solid-State Sensors and Actuators (Transducers '91), San Francusco, CA, USA, June 24-27, 1991, pp 22-25

6 C H Stephan and M Zanin1, A micromachined, stlicon mass-air-flow sensor for automotrve applications, Proc 6th Int Conf Soldd-State Sensors and Actuators (Transducers' 91), San Francisco, CA, USA, June 24-27, 1991, pp 30-33

7 B W van Oudheusden, Silicon thermal flow sensors, Sensors and Actuators A, 30 (1992) 5-26

8 R G Johnson and R G Higashi, A highly sensitive silicon chip microtransducer for air flow and differential pressure sensing applications, Sensors and Actuators, 11 (1987) 6372

9 M Esashi, Micro flow sensor and integrated magnetic oxygen sensor using it, Proc 6lh Int Conf Solud-State Sensors and Actuators (Transducers '91), San Francisco, CA, USA, June 24-27, 1991, pp 34-37

$10 N$ Swart, A Nathan, M Shams and $M$ Parameswaran, Numerical optimisation of flow-rate microsensors using circuit simulation tools, Proc 6th Int Conf Soltd-State Sensors and Actuators (Transducers '91), San Francisco, CA, USA, June $24-27,1991$, pp 26-29

11 T S J Lammerink, M Elwenspoek and J H J Fluitman, Integrated liquid dosing system, Prac MEMS '93 Conference, Fort Lauderdale, FL, USA, Feb 7-10, 1993, pp 254-259

12 R Miyake, T S J Lammerınk, M Elwenspoek and J H J Fluitman, Micro mixer with fast diffusion, Proc MEMS '93 Conference, Fort Lauderdale, FL, USA, Feb 7-10, 1993, pp $248-253$ 